\title{
EXPERIMENTAL CONTRIBUTION TO THE STUDY OF ANTISYPHILITIC HYPERTHERMY PRODUCED BY PHYSICAL AGENTS
}

By Professor J. F. A. ALBERT BESSEMANS, Director of the Institute of Hygiene and Bacteriology, University of Ghent (Belgium).

THE thermogenic procedures, which are used in combating diseases, can be classified in the manner shown by Table I.

The physiological methods are limited to animals. The infectious pathological procedures include induced malaria; the chemical ones the administration of vaccines. The physical procedures are those which we intend to examine here, so far as they are utilisable against syphilis.

Let us note that most of these procedures have, or may have, a double purpose, preventive and curative ; and a double action, local or general. They may have also a double biological mechanism, that is to say, a direct effect on the invading parasite and another effect on the infected host.

From a terminological standpoint, it should be remembered that the Latin word " febris " and the Greek word "puretos" mean "fever." Moreover, the Latin word " calor" (meaning originally " heat") as well as the Greek word " thermè " (originally " heat") and "pur" (originally " fire ") have been used to designate fever as well as heat, the "fire" accompanying it.

Springing from this etymology, the biological and medical literature has incorporated certain words with a definite meaning, such as febrigenic, apyretic, hyperthermia, and the like. But certain other words, applied 
Physiological (limited to animals) :

Brain puncture (rabbit).

Provocation of convulsions, etc.

Infectious pathological : inoculation of the germ of

Malaria.

Relapsing fever.

Sodoku (rat-bite fever).

Exanthemous typhus, etc.

Chemical: administration of

Cristalloids (nitrophenols).

Oil solutions (sulphurated oil).

Colloid salts (electrargol).

Heteroproteins (milk, gelatine, peptones, vaccines).

Physical :

Hot air.

Hot water and steam baths.

Hot paraffin.

Luminous and dark (infra-red) radiations.

Electric currents of high frequency :

With the electrodes in surface contact: medium waves (600-200 m.).

Without surface contact: short waves (30-10 m.), ultrashort waves (less than Io $\mathrm{m}$.).

\begin{tabular}{l|l|l}
$\begin{array}{l}\text { Double purpose : } \\
\begin{array}{l}\text { Preventive. } \\
\text { Curative. }\end{array}\end{array}$ & $\begin{array}{l}\text { Double action : } \\
\text { Local. } \\
\text { General. }\end{array}$ & $\begin{array}{l}\text { Double biological mechanism : } \\
\text { Direct effect on the germ. } \\
\text { Effect on the infected organism. }\end{array}$ \\
\hline
\end{tabular}

especially in reference to the artificial production of heat in living tissue, have caused regrettable misconceptions.

With the object of exchanging views which would tend toward simplifying and unifying the nomenclature, we suggest that all the words in which one or the other of the roots "febr" and "pyr" occur should apply only to "general " tissue heat. This being established, "fever," "pyrexia" and "general hyperthermy" would be synonymous; and the word "hyperpyrexia" having become redundant, could then be abandoned.

Depending on whether the purpose is preventive or therapeutic, the following terms would be used : general thermoprophylaxis (pyretoprophylaxis) and local thermoprophylaxis ; or general thermotherapy (pyretotherapy) and local thermotherapy. 
Table II.--Terminology Relating to Medical HYPERTHERMY

\begin{tabular}{l} 
Etymology \\
\hline Latin " febris" \\
Greek "puretos" $=$ FEVER \\
\hline Latin " calor" \\
Greek " "thermè "
\end{tabular}

Suggestion (since the roots "febr" and "pyr" apply only to "general" tissue heat).

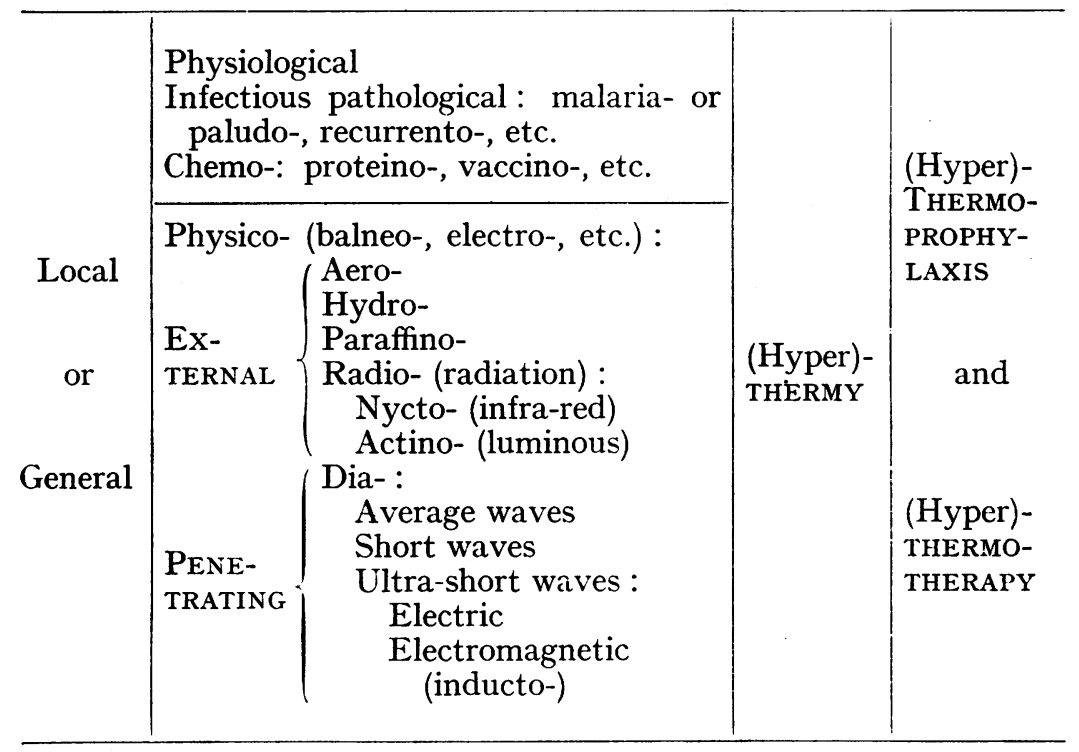

General (hyper)thermy $=$ pyrexia $=$ fever

General (hyper)thermoprophylaxis = pyretoprophylaxis

General (hyper)thermotherapy = pyretotherapy

Finally, as shown in Table II., different prefixes indicate either the agent employed to produce hyperthermy: malaria- or paludo-, recurrento-, proteino-, vaccino-, aero-, hydro, paraffino-, nycto- (infra-red), actino- (luminous), dia- (penetrating) ; or the thermogenic process : chemo-, physico-, balneo-, radio- (radiation), electro-, and so on. One would simply have to specify 
whether average, short or ultra-short waves are used, or electromagnetic induction (general inductothermy or inductopyrexia).

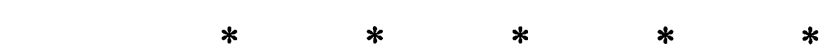

Hot baths were used by Schamberg and by us in treating animal syphilis. We were not aware of any previous work when we began our own studies in I926. Our curiosity was aroused because treponemata in cultures and emulsions were killed by temperatures which were compatible with life in man and in higher animals. As we learned later, Bronfenbrenner and Noguchi made analogous observations as early as I9I5.

We used in our experiments in the rabbit the syphilitic material obtained from the strains which we isolated in Ghent. Two years after these were isolated, their specific infectiousness was proven by an unfortunate accident. One of the assistants at our institute injected a slight quantity of an emulsion of a rabbit's syphiloma under the skin of his finger. He developed a secondary syphilitic eruption several weeks later, and his Wassermann reaction became positive.

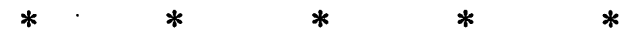

At first we employed general and local hot water baths in treating testicular syphilomata of rabbits. After this we treated similar lesions with hot air, obtained first from a Bunsen burner the heat from which was conducted through a curved chimney, and later from an electric hot air blower. About the same time, we also experimented with hot paraffin baths and packs. Luminous and infrared radiations were also used. Still later, medium, short and ultra-short wave radiations were employed. And then followed the use of a hot air chamber, employed alone or in conjunction with electromagnetic induction.

With most of these physical methods, one can produce a varying amount of local heat as well as a generalised temperature.

Since we are dealing primarily with temperatures, it is important to employ accurate temperature-measuring devices. These can be grouped, in general, as thermocouples and thermometers. Both have certain advantages, 
depending on the purpose for which they are to be used.

If local temperatures are to be measured in the absence of short and ultra-short waves, accurate data can be obtained by using a copper-constantan thermocouple, or, in certain cases, a Zondek depth thermometer. This latter fragile instrument fits into a thin metal cannula, which can be inserted into the depth of an organ.

When temperatures are measured in the electrical and electromagnetic high frequency fields, the current must be turned off if resistance pyrometers or thermocouples are employed. The same fact applies to mercury thermometers, except when placed within the body not too near to the electrodes. In any case this difficulty can be avoided by using quartz or even glass thermometers, filled with benzene or with mineral oil.

$$
\text { * } * * \quad * \quad * * \quad *
$$

By means of the various heating methods cited above and the use of the accurate temperature-measuring devices which have been described, we have established certain fundamental principles concerning the thermal death time in vivo of Treponema pallidum.

The first is that heating for one hour at $42^{\circ} \mathrm{C}$. $\left(\mathrm{IO} 7 \cdot 6^{\circ} \mathrm{F}\right.$.), two hours at $40^{\circ} \mathrm{C}$. (I04 ${ }^{\circ} \mathrm{F}$.), or several hours at a few tenths of a degree below this figure, usually suffices to bring about the immediate or gradual death of treponemata in testicular syphilomata of rabbits and in primary and secondary syphilitic lesions of man.

The action of such heat on the lesions is as follows. First, the micro-organism shows a loss or a weakening of its virulence, which can be proved by transfers into the testicle or on to the cornea of a fresh rabbit; and at the same time it becomes immobile. Then all treponemata disappear from the lesion. Finally, the lesion itself heals.

The rapidity with which these changes take place depends, as a rule, on the height of the temperature and the duration of its application.

Sometimes there is a late reaction to heat treatment, and the changes just outlined are observed only after several days. This is particularly true when no high and long-sustained treatment has been used. In this case we are clearly dealing with a systemic reaction of the animal.

The loss or the weakening of the virulence of the 75 
treponemata shows that the method has possibly some social prophylactic importance, because a chancre or a secondary lesion can be rapidly healed or deprived of its virulence by local as well as by general application of heat.

We have also shown that the heating of rabbit testicles soon after inoculation may prevent the appearance of a syphiloma and, in certain cases, even of a generalised infection. Nevertheless, further experiments must be performed in order to clarify this issue completely.

We have established a second fundamental fact: the treponemata in the popliteal lymph nodes of a syphilitic rabbit rarely lose their virulence if only the same amount of heat is applied in vivo as that which causes destruction of the treponemata of external lesions; it sometimes takes more than sixty minutes at $46^{\circ} \mathrm{C}$. (II $4 \cdot 8^{\circ} \mathrm{F}$.) to achieve the same result.

If the lymph nodes are not freed from virulent treponemata immediately after the application of heat, they may become sterile after several weeks. This is further evidence of the defensive reaction of the host.

It is possible that some other varieties of Treponema pallidum, modified by local conditions, are endowed in vivo with an analogous increase of thermo-resistance. We refer specially to the treponemata present in the testicle of the rabbit during the incubation period of the syphiloma, and to the treponemata found in the brain of the apparently non-syphilitic but truly infected mouse. These types seem to have about the same infectiousness as those found in the lymph nodes of the syphilitic rabbit.

There are some paradoxical facts which we have recently observed when heating testicular syphilomata of rabbits by means of medium damped or undamped diathermic waves of 200 to 300 metres.

Thus it sometimes happens in these cases that, even if temperatures which should be adequate for healing the lesion (according to our first principle) are employed, no 
effect is obtained. Occasionally also in the course of this treatment, if a lesion is cooled by air blowers to such an extent that (as the result of absence of hyperthermy) no healing should result, treponemata nevertheless disappear and the lesion heals after a few days.

These paradoxical reactions may possibly be explained, the first by a change in thermo-resistance of the microorganism, and both first and second by some contrary effects of high frequency currents on the neuro-vegetative system of the rabbit (analogous to the effects described in similar cases, under the influence of other factors, by Gastinel and Israelson and their associates) or by some other as yet unknown mechanisms.

The reaction of the whole organism becomes evident in these cases.

At present such observations are confined to the rabbit.

A few objections have been made to the fundamental principles I have summarised, and on which, in our opinion, antisyphilitic hyperthermy is based.

First: Levaditi and his collaborators treated syphilitic rabbit lesions and syphilitic mice in a short wave electromagnetic field. Their results were inconsistent; and from this they judged that individual factors accounted for these discrepancies. But they neglected to measure the tissue temperatures with accuracy, so that their interpretations do not seem to be very valuable.

Second: Giovanni Truffi reported that Treponema pallidum remains virulent when passed for one hour through the bodies of chickens and pigeons, which were supposed to have a body temperature of $42^{\circ} \mathrm{C}$.

Mario Truffi and Beck based certain theories on these results, and reached the unjustifiable conclusion that Treponema pallidum could resist, within the organism of birds, temperatures of $42^{\circ} \mathrm{C}$. for a period of at least five hours. They deduced from the experiments of Giovanni Truffi that the rise of temperature is not the sole cause of the action of malariatherapy and of pyretotherapy in general.

We believe that we have disproved these assumptions.

We have repeated the experiments of Giovanni Truffi, and have accurately measured the temperatures of various parts of the bodies of the animals during the 
experiments: Io hens, 3 roosters and 8 pigeons were used.

In the first place, the temperatures of various parts of the bodies of these animals vary greatly, in some instances from $35^{\circ}$ to $42^{\circ} \mathrm{C}$. Hence, if treponemata lodge in cool parts, they may retain their virulence.

Secondly, we believe that the experiments of Giovanni Truffi must have been carried out under peculiar conditions, because we were not able to confirm their results.

An emulsion of the blood, liver, brain and spinal cord of our 2I experimental infected birds failed to produce a single syphilitic lesion, when injected into the testicles of 42 rabbits who survived for at least three months; the popliteal lymph glands of these rabbits also remained sterile.

Furthermore, we must call attention to the fact that, as a syphilitic strain is passed through a series of rabbits, it gradually becomes more virulent for this animal. It is reasonable to assume that this is accompanied by an increased resistance of the strain to temperature.

Giovanni Truffi worked with a strain which had serially passed through the bodies of hundreds of rabbits since it was isolated about thirty years ago. As our own strains have aged they have become more virulent and in some cases seemingly more thermo-resistant.

An example of this is given by the following results. In I928 we were able to prevent the appearance of syphilomata or to cure those already present in rabbits in 7 out of $\mathrm{I} 3$ experiments, by simply transposing the testicles for several hours into the abdominal cavity. In I935 this was possible in only 3 out of 22 experiments, that is to say, in 13.5 per cent. of the cases instead of 53 per cent.

Third objection: Levaditi and his school have questioned the fact, which was first established by us, that the treponemata found in the popliteal lymph nodes of the rabbit have become thermo-resistant. These authors claim that because there is a reactionary inflammation of the testicular tissue which does not exist to an equal extent in the lymph nodes, the treponemata are destroyed by the tissue reaction to heat more easily in a syphiloma.

This theory has some interesting and attractive features. But we have shown that, when treponemata 


\section{DATA ON ANTISYPHILITIC HYPERTHERMIA}

have been removed from the lymph nodes, they immediately show a much greater virulence than those removed at the same time from the corresponding testicular lesions. Moreover, this argument of Levaditi and his followers, even if true, does not alter the fact that treponemata found in the lymph nodes of the rabbit are more thermo-resistant in vivo than those proliferating in the testicular syphilomata of the rabbit and in external lesions of early syphilis in man. In addition, Neymann, Lawless and Osborne have shown that treponemata persist in the lymph nodes of man after they have been destroyed in the primary lesions, provided the patient has been treated with high artificial fever alone for an extended period.

The only common factor of all methods of treating syphilis by means of disease, injections of protein or other foreign compounds, and by various physical methods, is heat.

Besides, it is well known that if a rabbit with a testicular syphiloma is immersed in a hot bath, given definite conditions of temperature and time, the syphiloma will disappear.

If the entire animal develops a sufficiently high fever as a result of the hot bath and if the infected testicle does not come in contact with the water, the syphiloma heals or not, according to whether it is fixed within the abdominal cavity, or is in a position where it is kept cool.

This disproves the absurd claim advanced many years ago, that water itself has an effect on the healing of syphilitic lesions. It shows that the favourable healing effects are caused by heat.

We have treated syphilitic rabbits by the " diathermax" apparatus (medium waves of I8 metres), the " inductotherm" (electro-magnetic waves of 25 metres) and the "ultrapandoros" (electric waves of 4 metres). The temperature of the syphilomata, of the popliteal lymph glands, of the surrounding air, of the mouth and of the rectum were measured, in the course of these experiments, with the benzene or mineral oil thermometers which have been described, without it being necessary to turn off the current.

There is, in such cases, a great variation of the tempera- 


\section{BRITISH JOURNAL OF VENEREAL DISEASES}

tures of the infected regions so measured, as long as they are not covered. These differences depend, of course, on the variability of the experimental conditions, especially of the temperature of the air in immediate contact with the regions studied.

But if, in analogous experiments, these regions are insulated against heat loss by covering them with cotton, high local temperatures can regularly be obtained.

Many animals die of heat stroke during such experiments. But if the temperature of a testicular syphiloma is raised to $42^{\circ} \mathrm{C}$. for one hour by this method, a destruction of the treponemata in the organ usually results. This is frequently not the case when the same temperature is produced in a popliteal lymph gland.

Thus, in our experience, the increased thermoresistance in vivo of the treponemata of the superficial lymphatic system of the syphilitic rabbit also exists against medium, short and ultra-short wave radiations. In other words, neither the wavelength nor the physical modality used has any effect per se on the killing of treponemata. The important factor is the temperature of the lesion.

We have proved in the same manner that in man the motility and virulence of the treponemata and the clinical appearance of chancres and mucous patches are unaffected if the local temperature is not high enough, whatever heating method is employed. The exposure of a lesion to an intense electric field, for instance, may not produce any changes, even if the intensity is so strong as to burn the superficial tissues.

We have also experimented in vitro with an emulsion of an Ehrlich sarcoma of the mouse, with various bacterial cultures, and with an emulsion of guinea-pig blood rich in trypanosomes.

If any of these materials are exposed to the action of short or ultra-short waves, sterilisation can be obtained. If, however, the test-tubes containing the products are cooled at the same time by means of air or water, sterilisation does not occur: provided, of course, the temperatures of the emulsions and cultures are kept low enough to prevent the thermal action of the electric waves.

Therefore, it is apparent that, in these experiments also, we are not dealing with any wave effect.

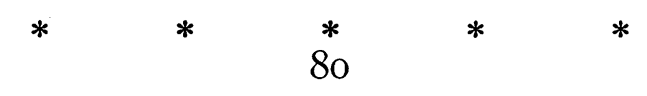


We have reported that there is a considerable variation of the temperature of different parts of the body of normal birds. This variation also exists in the organs and tissues of normal rabbits. It should be noted that there is ${ }^{\circ} \mathrm{a}$ variation of about $2 \frac{1}{2}^{\circ} \mathrm{C}$. in the temperatures of different parts of such a small organ as the eye of this animal; and that, even when hot baths are given to a rabbit, there is a variation of temperature in the various parts of the body.

Analogous observations may be made in man. In addition, during local diathermy of the deep organs of the true pelvis, the position of the active and of the inactive electrode has a decided influence on the temperature obtained in the cervix : this was proved by measurements made on a group of twelve women. Besides, similar variations according to the mode of application were observed in the abdominal cavity of the rabbit: this was accomplished by means of accurately calibrated thermocouples inserted through the anal opening.

All these experimental results prove the fundamental importance of the continuous measurement of tissue temperatures.

$* \quad * \quad * \quad * \quad * \quad * \quad *$

One of the most important elements, though not necessarily the sole factor of the malariatherapy of neurosyphilis, is undoubtedly heat.

Based on this theory, Neymann and Osborne began their work in producing artificial fever and treating neurosyphilis in I927. Their results with general paresis as well as those of other authors have confirmed this assumption. There is no difference, as far as end results are concerned, whether a paretic is treated with diathermy short undamped radio waves, or external heat, provided the patient attains a sufficient temperature and provided this is maintained for a period which is long enough.

Nevertheless, there are many inexplicable facts in the life-cycle of Treponema pallidum. It has been shown by Forster and by ourselves that treponemata found in the brain of paretics do not seem to be virulent for the rabbit. In I of 7 cases, where a piece of the brain substance of a living paretic was removed, we saw many mobile treponemata in the dark field. After an emulsion of this brain substance had been injected immediately into the 


\section{BRITISH JOURNAL OF VENEREAL DISEASES}

testicles of a number of rabbits no external lesion developed, and even the later transfers of the lymph nodes into a new series of rabbits remained negative. On the contrary, treponemata found in the brain of infected mice without apparent syphilis seem to be highly infectious.

It is not unlikely that other varieties of Treponema pallidum are modified by local conditions. Future research will have to elucidate this question. It is certain that we are dealing with a micro-organism of great adaptability and changeability. There are, therefore, many obscure biological factors which will have to be solved before the entire mechanism of antisyphilitic fever therapy is known, though this therapy is now a practical reality.

Following the idea of Simpson and Neymann that a judicious combination of chemo- and pyretotherapy might accomplish more than if either therapy is used alone, we devised a method by which rabbits could be treated with artificial fever and drugs.

A cabinet was constructed of wood, without the use of nails or metal parts. The animal was heated in this cabinet by hot air alone or combined with electromagnetic induction.

By careful technique in manipulation of the air temperature and the intensity of the magnetic field, the rabbit's fever could be maintained at approximately $42^{\circ} \mathrm{C}$. for half an hour. The rabbit is more sensitive to artificial fever than man, probably because of its deficiency in perspiration. It was, therefore, impossible to maintain the temperature in rabbits for as long a period as this has been possible in man.

Eleven rabbits survived. Six of these animals were treated with artificial fever alone and 5 with artificial fever and arsphenamine. For purposes of comparison, 5 other rabbits were treated with the same doses of the drug without fever.

The results may be summarised in the following manner. The lymph gland transfer into the testicles of a new series of rabbits was positive in 2 of the 5 animals treated only with arsphenamine. Two of the 6 rabbits treated with fever alone also gave positive transfers. As yet we have not been able to secure positive transfers 
from any of the 5 rabbits treated simultaneously with fever and arsphenamine.

We have not employed serological tests for our rabbits, because, as has been conclusively shown in our previous publications, rabbits when infected with the pallidoid treponema, that is to say, Spirocheta cuniculi, may give positive serological tests. Syphilitic rabbits may give negative serological tests and normal ones may even give positive reactions. Serological tests of the blood serum of rabbits are therefore regarded as valueless in practice.

\section{Conclusions}

(I) The various methods for producing temperatures in animals and in man have been reviewed, and we have especially emphasised that a thermometer made of quartz or even of glass and filled with benzene or mineral oil can accurately be employed for the measurement of temperatures in the high frequency fields. Such thermometers are not affected by the electric nor by the electromagnetic waves.

(2) It has been shown that treponemata found in the testicular syphilomata of rabbits and in the external lesions of early syphilis in man are thermo-labile in vivo in a definite temperature time interval. Treponemata found in the lymph nodes of syphilitic rabbits are more virulent and thermo-resistant. In the latter case the thermo-resistance is so great that it surpasses that of the tissues of man and animals.

(3) The objections made to these fundamental principles have been disproved.

(4) Paradoxical and unexplained results after treatment with diathermy occasionally occur in rabbits. This is attributable to the reaction of the animal organism as a whole.

(5) The healing effect of hyperthermy applied to syphilis is in part at least purely a matter of temperature. This healing effect must be considered in the light of the temperature variations in various parts of the animal body.

(6) The position of the active and of the inactive electrode during local diathermy of the abdominal organs, in the rabbit and in man, has a decided effect on the deep temperatures reached. 
(7) The type of heating, such as the wavelength of an electromagnetic current, has nothing whatever to do with any secondary treponemicidal effect of these currents. These secondary effects are merely produced by heating of the tissues or media in which treponemata are found.

(8) If bacterial cultures, treated in vitro with intense short or ultra-short wave radiation, are cooled so that no heating effect results, they are not affected. The same fact applies to emulsions of the Ehrlich sarcome of the mouse and of trypanosomes.

(9) Treponemata found in the brain of paretics seem to have no virulence for the rabbit, while those found in the brain of the syphilitic mouse seem to be very virulent. The basic reasons for these facts are as yet unknown.

(Io) There are modified varieties of Treponema pallidum which develop in the course of its life-cycle. This adaptability of the micro-organism renders the cure of syphilis difficult.

(II) A judicious use of the combination of chemo- and pyretotherapy seems to be more effective in the syphilitic rabbit than when either therapy is used alone. Serological tests for syphilis are valueless criteria for judging the presence, progress, or the persistence of the disease in rabbits. 